Noname manuscript No.

(will be inserted by the editor)

\title{
Convergence Analysis of Processes with Valiant Projection Operators in Hilbert Space
}

Yair Censor ${ }^{1}$ and Rafiq Mansour ${ }^{2}$

December 4, 2016. Revised: May 29, 2017. Revised: September 11, 2017.

\begin{abstract}
Convex feasibility problems require to find a point in the intersection of a finite family of convex sets. We propose to solve such problems by performing set-enlargements and applying a new kind of projection operators called valiant projectors. A valiant projector onto a convex set implements a special relaxation strategy, proposed by Goffin in 1971, that dictates the move toward the projection according to the distance from the set. Contrary to past realizations of this strategy, our valiant projection operator implements the strategy in a continuous fashion. We study properties of valiant projectors and prove convergence of our new valiant projections method. These results include as a special case and extend the 1985 automatic relaxation method of Censor.
\end{abstract}

Keywords Intrepid projector; convex feasibility problem; valiant projector; set-enlargement; automatic relaxation method (ARM); ART3 algorithm; Goffin's principle

Mathematics Subject Classification (2000) 65K05, 90C25

\section{Introduction}

We consider the convex feasibility problem (CFP) in a real Hilbert space. It consists of a finite family of closed and convex sets with a nonempty intersection and calls to find an element in this intersection. There are many algorithms in the literature for solving CFPs, see, e.g., 1,2, and many problems in operations research and in various other fields can be presented as feasibility problems. For example, a problem of road design is modeled as a feasibility problem in [3, where the motivation is automated design of road alignments. A road alignment is represented by the center-line of the road, which is idealized as a (generally) nonlinear, smooth curve. To facilitate construction drawings, civil engineers reduce the three-dimensional road design to two two-dimensional parts, horizontal and vertical. For a new approach to road design

\footnotetext{
${ }^{1}$ Corresponding Author. Department of Mathematics, University of Haifa, Mt. Carmel, Haifa 3498838, Israel. Email: yair@math.haifa.ac.il. ${ }^{2}$ Department of Mathematics, University of Haifa, Mt. Carmel, Haifa 3498838, Israel. E-mail: intogral@gmail.com.
} 
optimization see [4]. In [5] the authors give general recommendations for successful application of the Douglas-Rachford feasibility-seeking method to convex and non-convex real matrix-completion problems. The work in [6] focuses on the problem of protein conformation determination formulated within the framework of matrix completion by solving CFPs. In [7, section 6] solving Sudoku puzzles is modeled as an integer feasibility problem. The books [8, [9] and [10] contain many algorithms and methods that solve the CFP, and there is also a description of a wide range of operators and their properties. Hence, the importance of the CFP stems from its flexibility to accommodate problems from various fields and simplify their understanding and solutions.

Searching for a solution to a system of linear equations is a convex feasibility problem and has led to many different iterative methods. When the system of linear equations is inconsistent, due to modeling or measurements inaccuracies, it has been suggested to replace it by a system of pairs of opposing linear inequalities, that represent nonempty hyperslabs. Applying projection methods to this problem can be done by using any iterative method for linear inequalities, such as the method of Agmon [1] and Motzkin and Schoenberg 12 (AMS). However, in order to improve computational efficiency, Goffin 13 proposed to replace projections onto the hyperslabs by a strategy of projecting onto the original hyperplane (from which the hyperslab was created), when the current iterate is "far away" from the hyperslab, and reflecting into the hyperslab's boundary, when the current iterate is "close to the hyperslab", while keeping the iterate unchanged if it is already inside the hyperslab.

In 14] Herman suggested to implement Goffin's strategy by using an additional enveloping hyperslab in order to determine the "far" and the "close" distance of points from the hyperplane, resulting in his "Algebraic Reconstruction Technique 3" (ART3) algorithm. In [15] Censor also embraced the idea of hyperslabs, and defined an algorithmic operator that implemented Goffin's strategy in a continuous manner, resulting in the Automatic Relaxation Method (ARM). For applications and additional details see [16] and [17.

A fundamental question, that remained open since then, was whether the hyperslabs approach to handle linear equations and Goffin's principle can be applied to general convex sets and not only to linear equations. This question was recently studied by Bauschke, Iorio and Koch in [18, see also [3] and 4 , for further details and interesting applications. They defined convex sets enlargements instead of hyperslabs and used them to generalize the algorithmic operator that appeared in [14]. They defined an operator which they called the intrepid projector, intended to generalize the ART3 algorithm of [14] to convex sets. Motivated by 18, we present in this paper a new operator which we call the valiant operator, that enables to implement the algorithmic principle embodied in the ARM of [15] to general convex feasibility problems. Observe that both ART3 and ARM seek a feasible point in the intersections of the hyperslabs and so their generalizations to the convex case seek feasibility of appropriate enlargement sets that define the extended problem. 
The new valiant projection method (VPM) proposed and studied here answers affirmatively the theoretical question posed above. To date we have no computational experience with it, that will allow us make any claims about its actual advantages. It may be the case that using valiant operators is beneficial not always but only in some specific situations (size, sparsity, nature of problems, parameters, specific applications, etc.) or under some additional conditions. To discover these a methodological numerical work is required and we plan to undertake such work with collaborators from some application fields with which we are involved.

The paper is organized as follows: In Section 2 we give definitions and preliminaries. In Section 3 we present the motivation of the main idea of this paper and in Section 4 we present the new algorithmic projector and its relevant features. Finally, in Section 5. we present our new algorithm and prove its convergence, and in Section 6 we offer concluding comments.

\section{Preliminaries}

For the reader's convenience we include in this section some properties of operators in Hilbert space that will be used to prove our results. We use the recent excellent book of Cegielski [9] as our desk-copy in which all the results of this section can be found [9, Chapter 2 and Chapter 3]. Let $\mathcal{H}$ be a real Hilbert space with inner product $\langle\cdot, \cdot\rangle$ and induced norm $\|\cdot\|$, and let $X \subseteq \mathcal{H}$ be a closed convex subset. Denote the index set $I:=\{1,2, \ldots, m\}$. If $\Omega \subseteq \mathcal{H}$ and $x \in \mathcal{H}$ then we denote by $P_{\Omega}(x)$ the metric projection of $x$ onto $\Omega$.

Definition 2.1 An operator $T: X \rightarrow \mathcal{H}$ is:

i. Nonexpansive (NE), if $\|T(x)-T(y)\| \leq\|x-y\|$ for all $x, y \in X$.

ii. Firmly nonexpansive (FNE), if $\|T(x)-T(y)\|^{2}+\|(x-T(x))-(y-T(y))\|^{2} \leq\|x-y\|^{2}$ for all $x, y \in X$.

Remark 2.1 (i) It is clear from Definition 2.1 that every FNE operator is NE. See also, 9, Theorem 2.2.10 (i)-(ii)]. (ii) By [9, Lemma 2.1.12] the family of NE is closed under convex combinations and compositions.

Definition 2.2 Let $C$ be a nonempty closed convex subset of $\mathcal{H}$, let $x \in \mathcal{H}$, and let $c \in C$. Denote the distance from $x$ to $C$ by $d_{C}(x):=\inf _{c \in C}\|x-c\|$, the infimum is attained at a unique vector called the projection of $x$ onto $C$ and denoted by $P_{C}(x)$.

Proposition 2.1 [10, Proposition 4.8] Let B be a nonempty closed convex subset of $\mathcal{H}$. Then the projector $P_{B}$ is FNE.

Definition 2.3 An operator $T: X \rightarrow \mathcal{H}$ having a nonempty fixed point set Fix $T=\{x \in X \mid T(x)=x\}$ is:

i. Quasi-nonexpansive (QNE) if $\|T(x)-z\| \leq\|x-z\|$ for all $x \in X$ and $z \in$ FixT. 
ii. Strictly quasi-nonexpansive (sQNE) if $\|T(x)-z\|<\|x-z\|$ for all $x \notin$ Fix $T$ and $z \in$ Fix $T$.

iii. $B$-strictly quasi-nonexpansive ( $B$-sQNE), where $B \neq \varnothing$ and $B \subseteq$ Fix $T$, if $T$ is quasi-nonexpansive and $\|T(x)-z\|<\|x-z\|$ for all $x \notin \mathrm{Fix} T$ and $z \in B$.

iv. $\alpha$-strongly quasi-nonexpansive ( $\alpha$-SQNE) if $\|T(x)-z\|^{2} \leq\|x-z\|^{2}-\alpha\|T(x)-x\|^{2}$ for all $x \in X$ and $z \in \operatorname{Fix} T$, where $\alpha \geq 0$. If $\alpha>0$ then $T$ is called strongly quasi-nonexpansive (SQNE).

The next implications follow directly from the definitions, see 9, page 47] and 9, Remark 2.1.44(iii)].

Proposition 2.2 For an operator $T: X \rightarrow \mathcal{H}$ having a fixed point, the following statements hold:

i. If $T$ is sQNE then $T$ is $B$-sQNE, where $B \subseteq$ FixT.

ii. If $T$ is FixT-sQNE then $T$ is $s Q N E$.

iii. If $T$ is $S Q N E$ then it is $s Q N E$.

The following proposition presents the relationship between NE and QNE operators.

Proposition 2.3 9, Lemma 2.1.20] An NE operator $U: X \rightarrow \mathcal{H}$ with a fixed point is $Q N E$.

Remark 2.2 From [9, Corollary 2.1.47] and [9, Fig. 2.14], a family of SQNE operators with a common fixed point is closed under convex combinations and compositions.

The following proposition shows that the relaxation of a projection onto a nonempty closed convex set is an SQNE operator.

Proposition 2.4 [18, Fact 1] Let $\Omega$ be nonempty closed convex subset of $\mathcal{H}$ and let $\lambda \in] 0,2[$. Set $R:=$ $(1-\lambda) \operatorname{Id}+\lambda P_{\Omega}$, let $x \in \mathcal{H}$ and $c \in \Omega$. Then

$$
\|x-c\|^{2}-\|R(x)-c\|^{2} \geq \frac{2-\lambda}{\lambda}\|x-R(x)\|^{2} .
$$

Theorem 2.1 [9, Theorem 2.1.26(ii)] Let the operators $U_{i}: X \rightarrow X, i \in I$, with $\bigcap_{i \in I}$ Fix $U_{i} \neq \varnothing$, be $B$-sQNE, where $B \subseteq \bigcap_{i \in I} \operatorname{Fix} U_{i}, B \neq \varnothing$. If $U:=U_{m} U_{m-1} \ldots U_{1}$ then

$$
\operatorname{Fix} U=\bigcap_{i \in I} \operatorname{Fix} U_{i}
$$

and $U$ is $B-s Q N E$.

Definition 2.4 An operator $U: X \rightarrow X$ is asymptotically regular if for all $x \in X$,

$$
\lim _{k \rightarrow \infty}\left\|U^{k+1}(x)-U^{k}(x)\right\|=0
$$

Theorem 2.2 9], Theorem 3.4.3] Let $U: X \rightarrow X$ be an operator with a fixed point. If $U$ is $S Q N E$ then it is asymptotically regular. 
The following well-known theorem is due to Opial.

Theorem 2.3 [9, Theorem 3.5.1] Let $X \subseteq \mathcal{H}$ be a nonempty closed convex subset of a Hilbert space $\mathcal{H}$ and let $U: X \rightarrow X$ be a nonexpansive and asymptotically regular operator with a fixed point. Then, for any $x \in X$, the sequence $\left\{U^{k}(x)\right\}_{k=0}^{\infty}$ converges weakly to a point $z \in \mathrm{Fix} U$.

Definition 2.5 An operator $T: X \rightarrow \mathcal{H}$ is demiclosed at 0 if for any weakly convergent sequence $x^{k} \rightarrow$ $y \in X$ with $T\left(x^{k}\right) \rightarrow 0$ we have $T(y)=0$.

The next theorem is known as the demiclosedness principle.

Theorem 2.4 9, Lemma 3.2.5] Let $T: X \rightarrow \mathcal{H}$ be an $N E$ operator and $y \in X$ be a weak cluster point of a sequence $\left\{x^{k}\right\}_{k=0}^{\infty}$. If $\left\|T\left(x^{k}\right)-x^{k}\right\| \longrightarrow 0$, then $y \in \operatorname{Fix} T$.

The following definition extends Definition 2.4 to a sequence of operators.

Definition 2.6 Let $X \subseteq \mathcal{H}$ be a nonempty closed convex subset. We say that a sequence of operators $U_{k}: X \rightarrow X$ is asymptotically regular, if for any $x \in X$

$$
\lim _{k}\left\|U_{k} U_{k-1} \ldots U_{0}(x)-U_{k-1} \ldots U_{0}(x)\right\|=0
$$

or, equivalently,

$$
\lim _{k}\left\|U_{k}\left(x^{k}\right)-x^{k}\right\|=0
$$

where the sequence $\left\{x^{k}\right\}_{k=0}^{\infty}$ is generated by recurrence $x^{k+1}=U_{k}\left(x^{k}\right)$ with $x^{0}=x$.

Theorem 2.5 9, Theorem 3.6.2(i)] Let $X \subseteq \mathcal{H}$ be a nonempty closed convex subset, $S: X \rightarrow \mathcal{H}$ be an operator with a fixed point and such that $S-\operatorname{Id}$ is demiclosed at 0 . Let $\left\{U_{k}\right\}_{k=0}^{\infty}$ be an asymptotically regular sequence of quasi-nonexpansive operators $U_{k}: X \rightarrow X$ such that $\bigcap_{k=0}^{\infty} \operatorname{Fix} U_{k} \supseteq \operatorname{Fix} S$. Let the sequence $\left\{x^{k}\right\}_{k=0}^{\infty}$ be generated by recurrence $x^{k+1}=U_{k}\left(x^{k}\right)$, with an arbitrary $x^{0} \in X$. If the sequence of operators $\left\{U_{k}\right\}_{k=0}^{\infty}$ has the property

$$
\lim _{k}\left\|U_{k}\left(x^{k}\right)-x^{k}\right\|=0 \Longrightarrow \lim _{k}\left\|S\left(x^{k}\right)-x^{k}\right\|=0,
$$

then $\left\{x^{k}\right\}_{k=0}^{\infty}$ converges weakly to a point $z^{*} \in \operatorname{Fix} S$.

\section{The Valiant Projector: Intuition and Motivation}

In this section, we explain the motivation and intuition behind valiant projection operators which relies on two basic ideas: the notion of enlargement of a convex set, and a strategy proposed by Goffin [13] that dictates to move towards the projection according to the distance to the set. In more details, the development was as follows. First, Herman proposed and studied in [14] the "Algebraic Reconstruction 
Technique 3" (ART3) algorithm for solving a system of two-sided linear inequalities. He set out to solve a large and sparse, possibly inconsistent, system of linear equations stemming from the problem of image reconstruction from projections and replaced each equation by a pair of opposing half-spaces yielding a consistent system of hyperslabs. Instead of applying to the system any available projection method he created around each hyperslab an additional wider enveloping hyperslab in order to implement a relaxation strategy of Goffin [13] that advocated interlacing steps of projection onto the hyperslab's median hyperplane with reflections into the bounding hyperpalnes of the hyperslabs. Secondly, Bauschke, Iorio and Koch proposed in [18] an operator, which they called the intrepid projector, for extending the ART3 method to handle convex sets in Hilbert space. They replaced the hyperslabs by enlargements of convex sets.

We consider the Automatic Relaxation Method (ARM) of [15] which implemented the strategy of Goffin in a continuous manner, without using additional enveloping hyperslabs to define whether a point is "close" or "far" from a hyperslab, as in ART3. The extension of ART3 from linear hyperslabs to general convex sets by Bauschke, Iorio and Koch is our inspiration in the present work. We generalize the ARM algorithm to encompass enlargements of convex sets instead of being limited to handle only systems of linear hyperslabs.

Enlargements of convex sets are defined as follows.

Definition 3.1 18, Definition 2] Given a nonempty closed convex subset $C$ of a Hilbert space $\mathcal{H}$, and $\alpha \geq 0$, the set

$$
C_{[\alpha]}:=\left\{x \in \mathcal{H} \mid d_{C}(x) \leq \alpha\right\}
$$

is the $\alpha$-enlargement of $C$.

Full details about ART3 and about Bauschke, Iorio and Koch's algorithm can be found in their papers. For the readers' convenience we give here only a brief account. In ART3, in addition to the construction of hyperslabs and enveloping hyperslabs, the ART3 makes three possible iterative steps: the projection step, the identity step and the reflection step. The location of a current iterate $x^{k}$ determines the appropriate step to be taken. If $x^{k}$ is outside the enveloping additional hyperslab then it is considered to be "far" from the original hyperplane, and, accordingly, a projection step onto the original hyperplane (which is the median of the hyperslab) will be taken. If $x^{k}$ is inside the first hyperslab then ART3 will keep it unchanged. But, if $x^{k}$ is located in the enveloping hyperslab then it is considered to be "near" the original hyperplane and it will be reflected into the boundary of the hyperslab. 


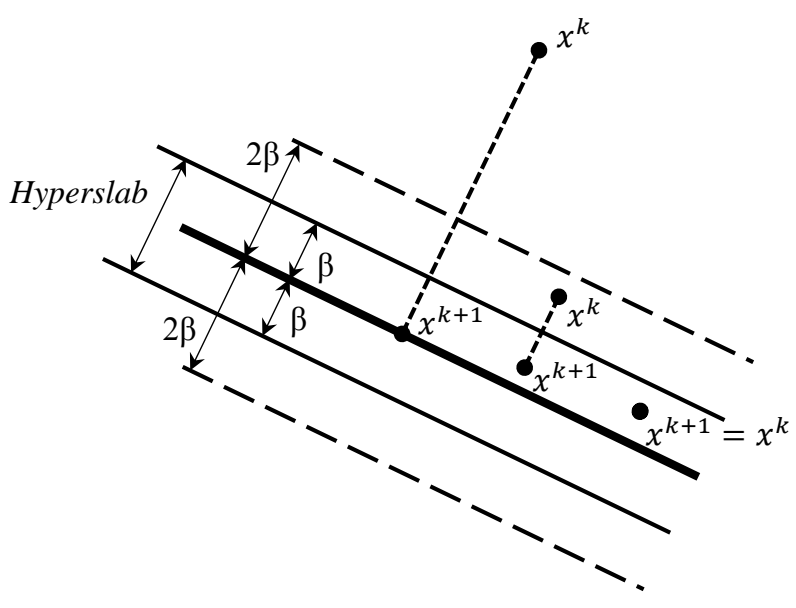

Fig. 1 The possible iterative steps of ART3.

Turning to the work of Bauschke, Iorio and Koch, the operator that accurately conveys the above ART3 algorithmic strategy to deal with convex sets is the following operator which we call here the "geometric intrepid projector".

Definition 3.2 (The geometric intrepid projector) Let $Z$ be a nonempty closed convex subset of $\mathcal{H}$, let $\beta \in \mathbb{R}, \beta>0$ and set $C:=Z_{[\beta]}$. The geometric intrepid projector $G_{C}: \mathcal{H} \rightarrow \mathcal{H}$ onto $C$ (with respect to $Z$ and $\beta$ ) is defined by

$$
G_{C}(x):= \begin{cases}P_{Z}(x), & \text { if } d_{Z}(x) \geq 2 \beta \\ x, & \text { if } d_{Z}(x) \leq \beta \\ x+2\left(\frac{\beta}{d_{Z}(x)}-1\right)\left(x-P_{Z}(x)\right), & \text { otherwise. }\end{cases}
$$

The properties of 8 for general convex sets remain to be investigated but we care to present the geometric intrepid projector $G_{C}$ because Bauschke, Iorio and Koch defined in [18] a different intrepid projector which does not capture precisely the ART3 strategy, and which we will name here the "BIK intrepid projection". Contrary to [18, Example 5], the third line in (9) which is supposed to mimic the reflection step of ART3, does not actually do so.

\section{Definition 3.3 [18, Definition 4] (The Bauschke, Iorio and Koch (BIK) intrepid projector)}

Let $Z$ be a nonempty closed convex subset of $\mathcal{H}$, let $\beta \in \mathbb{R}, \beta \geq 0$ and set $C:=Z_{[\beta]}$. The projector $Q_{C}: \mathcal{H} \rightarrow \mathcal{H}$ onto $C$ (with respect to $Z$ and $\beta$ ), henceforth called the BIK intrepid projector, is defined by [for all positive $\beta$ ] 


$$
Q_{C}(x):= \begin{cases}P_{Z}(x), & \text { if } d_{Z}(x) \geq 2 \beta, \\ x, & \text { if } d_{Z}(x) \leq \beta, \\ x+\left(1-\frac{d_{Z}(x)}{\beta}\right)\left(x-P_{Z}(x)\right), & \text { otherwise. }\end{cases}
$$

Although it is reasonable to define an algorithm like Bauschke, Iorio and Koch's algorithm but with $G_{C}$ instead of $Q_{C}$ we do not stop to do so here and proceed, in the next section, directly to our valiant projector and its properties.

\section{The Valiant Projector}

In this section we define the valiant projector in Hilbert space and study its properties.

Definition 4.1 (The valiant projector) Let $Z$ be a nonempty closed convex subset of $\mathcal{H}$, let $\beta \in \mathbb{R}$, $\beta>0$ and set $C:=Z_{[\beta]}$. The valiant projector $V_{C}: \mathcal{H} \rightarrow \mathcal{H}$, onto $C$ (with respect to $Z$ and $\beta$ ) is defined by

$$
V_{C}(x):= \begin{cases}x, & \text { if } d_{Z}(x) \leq \beta \\ x+\frac{\tau}{2}\left(1-\left(\frac{\beta}{d_{Z}(x)}\right)^{2}\right)\left(P_{Z}(x)-x\right), & \text { otherwise }\end{cases}
$$

with $\tau \in] 0,2[1$

The valiant projector works as follows: If the distance of a point $x$ from the set $Z$ is less than the depth $\beta$ of the enlargement, i.e., the point is inside the enlargement, then the operator leaves the point unchanged. Otherwise, if the distance of the point from the set is greater than the depth of the enlargement, then the valiant projector brings the point closer to the set $Z$ in the orthogonal projection direction of the point onto the set. From the term $1-\left(\frac{\beta}{d_{Z}(x)}\right)^{2}$ we see that the farther the point is from the set, the operator will progress towards the set in larger steps. Like in [15], the overall step-size also incorporates an additional user-chosen relaxation parameter $\tau$.

The valiant projector has the following useful property.

Proposition 4.1 Let $Z$ be a nonempty, closed and convex subset of $\mathcal{H}$, let $\beta \in \mathbb{R}, \beta>0$. If $C:=Z_{[\beta]}$ then the valiant projector $V_{C}$ of $(10)$ is SQNE.

Proof We prove that $V_{C}$ is SQNE with respect to $C$ and $\beta$. For the case $d_{Z}(x) \leq \beta$ we have $V_{C}(x)=x$ and the SQNE of $V_{C}$ is trivial. For the case $d_{Z}(x)>\beta$, the operator $V_{C}$ can be written as

$$
V_{C}(x)=\left(1-\frac{\tau}{2}\left(1-\left(\frac{\beta}{d_{Z}(x)}\right)^{2}\right)\right) x+\frac{\tau}{2}\left(1-\left(\frac{\beta}{d_{Z}(x)}\right)^{2}\right) P_{Z}(x),
$$

\footnotetext{
1 We keep the $\frac{\tau}{2}$ in the formula just to show its similarity with the ARM operator of [15].
} 
with $\tau \in] 0,2[$. Let

$$
\gamma(x):=\frac{\tau}{2}\left(1-\left(\frac{\beta}{d_{Z}(x)}\right)^{2}\right)
$$

then we have

$$
V_{C}(x)=(1-\gamma(x)) x+\gamma(x) P_{Z}(x)
$$

with $\gamma(x) \in] 0,1\left[\right.$. Using Proposition 2.4 with $\Omega=Z, \lambda=\gamma(x)$ and $R=V_{C}$ we have, for all $c \in Z$,

$$
\|x-c\|^{2}-\left\|V_{C}(x)-c\right\|^{2} \geq \frac{2-\gamma(x)}{\gamma(x)}\left\|x-V_{C}(x)\right\|^{2} \geq\left\|x-V_{C}(x)\right\|^{2} .
$$

Thus, $V_{C}$ is 1-SQNE therefore, SQNE, by Definition 2.3(iv).

Since both the identity operator Id and the projection $P_{Z}$ are NE, any convex combination of them will be also NE. However, the dependence of $\gamma$ in (13) on $x$ requires special attention when attempting to show that the valiant operator of 13 is NE. This is done in the next proposition.

Proposition 4.2 Let $Z$ be a nonempty closed convex subset of $\mathcal{H}$, let $\beta \in \mathbb{R}, \beta>0$. If $C:=Z_{[\beta]}$ then the valiant projector $V_{C}$ is $N E$.

Proof The proof is split into the three possibilities that have to be considered according to whether $V_{C}$ realizes the first or the second line of its definition $(10)$.

Possibility A: The first line of $\sqrt{10}$ holds for both points $x$ and $y$. In this case $d_{Z}(x) \leq \beta$ and $d_{Z}(y) \leq \beta$ thus, $V_{C}(x)=x$ and $V_{C}(y)=y$ so that $V_{C}$ is trivially NE.

Possibility B: The second line of 100 holds for both points $x$ and $y$. Without loss of generality, take any two points $x, y \in \mathcal{H}$ such that

$$
\left\|P_{Z}(y)-y\right\| \leq\left\|P_{Z}(x)-x\right\|,
$$

and denote

$$
\begin{aligned}
& d_{Z}(x)=\left\|P_{Z}(x)-x\right\|=a \beta, \\
& d_{Z}(y)=\left\|P_{Z}(y)-y\right\|=b \beta,
\end{aligned}
$$

for some real $1<b \leq a$. Then

$$
\frac{\left\|V_{C}(x)-x\right\|}{\left\|V_{C}(y)-y\right\|}=\left(\frac{1-\frac{1}{a^{2}}}{1-\frac{1}{b^{2}}}\right) \frac{\left\|P_{Z}(x)-x\right\|}{\left\|P_{Z}(y)-y\right\|},
$$


so that

$$
\left\|V_{C}(y)-y\right\| \leq\left\|V_{C}(x)-x\right\|
$$

There are three possible locations of the points $x, y, V_{C}(x)$ and $V_{C}(y)$ with respect to the set $Z$, see, Figure 2

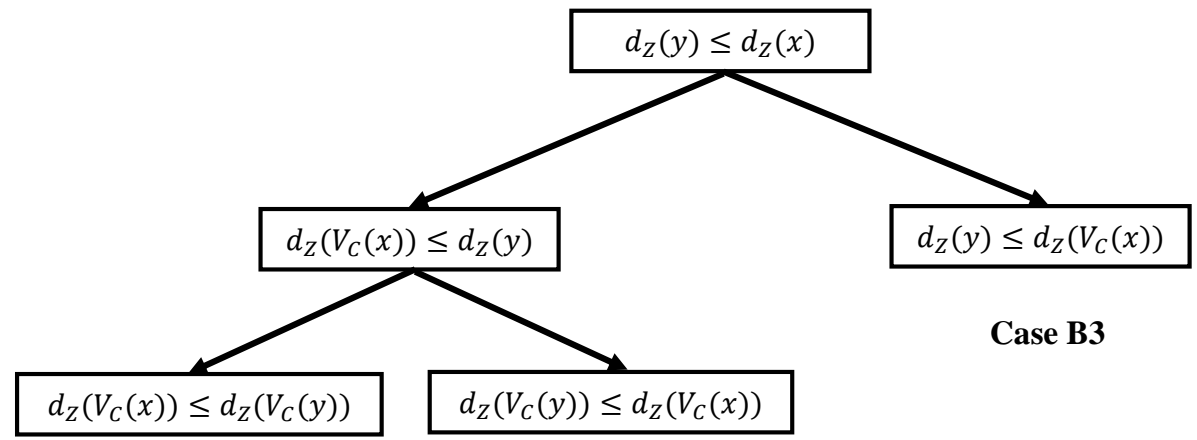

Case B1

Case B2

Fig. 2 Cases for Possibility B in the proof of Proposition 4.2

Below we discuss each case separately.

Case B1. Assume that

$$
\left\|P_{Z}\left(V_{C}(x)\right)-V_{C}(x)\right\| \leq\left\|P_{Z}\left(V_{C}(y)\right)-V_{C}(y)\right\| .
$$

This implies that

$$
\left\|P_{Z}(x)-V_{C}(x)\right\| \leq\left\|P_{Z}(y)-V_{C}(y)\right\|,
$$

because $P_{Z}\left(V_{C}(x)\right)=P_{Z}(x)$ and $P_{Z}\left(V_{C}(y)\right)=P_{Z}(y)$ which follows from the fact that $x, V_{C}(x)$ and $P_{Z}\left(V_{C}(x)\right)$ lie on the same line, and similarly for the other equality, see Figure 3

To study this case we add two enlargements to the set $Z$, one with a width of $\left\|P_{Z}(y)-y\right\|$ and the other with a width of $\left\|P_{Z}(y)-V_{C}(y)\right\|$. We denote the intersection point of the line through $x$ and $P_{Z}(x)$ with the boundary of the first enlargement by $\hat{y}$. Therefore, the intersection of the above mentioned line with the boundary of the second enlargement is exactly $V_{C}(\hat{y})$. This is so because, by 10 , points which are at the same distance from $Z$ have their images under a valiant operator also at equal distances from $Z$. See Figure 3, where the dashed lines are the enlargements. 


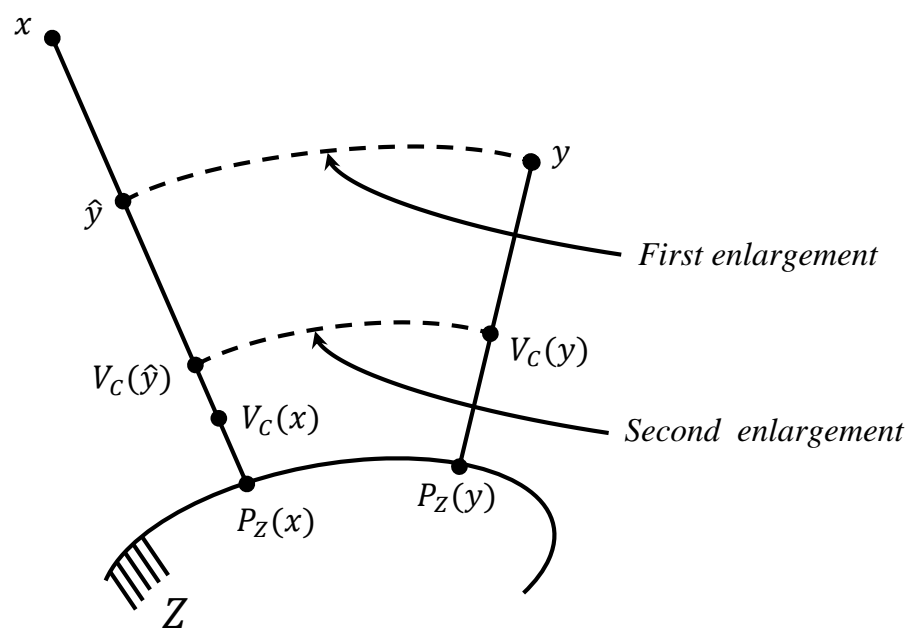

Fig. 3 Case B1 in the proof of Proposition 4.2

Now we calculate the relevant distances. First, note that

$$
\begin{aligned}
\|x-\hat{y}\| & =\left\|P_{Z}(x)-x\right\|-\left\|P_{Z}(x)-\hat{y}\right\| \\
& =\left\|P_{Z}(x)-x\right\|-\left\|P_{Z}(y)-y\right\| \\
& =a \beta-b \beta \\
& =(a-b) \beta .
\end{aligned}
$$

Secondly,

$$
\begin{aligned}
\left\|V_{C}(x)-V_{C}(\hat{y})\right\| & =\left\|P_{Z}(y)-V_{C}(y)\right\|-\left\|P_{Z}(x)-V_{C}(x)\right\| \\
& =\left\|P_{Z}(y)-y\right\|-\left\|V_{C}(y)-y\right\|-\left(\left\|P_{Z}(x)-x\right\|-\left\|V_{C}(x)-x\right\|\right) \\
& =b \beta-\left|\frac{\tau}{2}\left(1-\left(\frac{\beta}{b \beta}\right)^{2}\right)\right|\left\|P_{Z}(y)-y\right\|-a \beta+\left|\frac{\tau}{2}\left(1-\left(\frac{\beta}{a \beta}\right)^{2}\right)\right|\left\|P_{Z}(x)-x\right\| \\
& =b \beta-\frac{\tau}{2}\left(1-\frac{1}{b^{2}}\right) b \beta-a \beta+\frac{\tau}{2}\left(1-\frac{1}{a^{2}}\right) a \beta=\beta(a-b)\left(\frac{\tau}{2}\left(1+\frac{1}{a b}\right)-1\right) .
\end{aligned}
$$

Since, by the definitions of the parameters $a, b$ and $\tau$,

$$
\frac{\tau}{2}\left(1+\frac{1}{a b}\right)-1<1
$$

we have, by 22 , that

$$
\left\|V_{C}(x)-V_{C}(\hat{y})\right\| \leq \beta(a-b)=\|x-\hat{y}\| .
$$


Since the enlargement of a convex set is also a convex set,

$$
V_{C}(y)=P_{Z_{\left[\left\|P_{Z}(y)-V_{C}(y)\right\|\right]}}(y)
$$

and

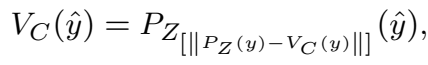

and

$$
\hat{y}=P_{Z_{\left[P_{Z}(y)-y \|\right]}}(x) .
$$

As is well-known, the metric projection is NE, so, by 26 and 27,

$$
\left\|V_{C}(y)-V_{C}(\hat{y})\right\| \leq\|y-\hat{y}\|
$$

By the characterization of the metric projection, see, e.g., [9, Theorem 1.2.4], for $P_{Z_{\left[\left\|P_{Z}(y)-y\right\|\right]}}(x)$ we have

$$
\langle x-\hat{y}, y-\hat{y}\rangle \leq 0
$$

and for $P_{Z_{\left[\left\|P_{Z}(y)-V_{C}(y)\right\|\right]}}(\hat{y})$ we have

$$
\left\langle x-V_{C}(\hat{y}), V_{C}(y)-V_{C}(\hat{y})\right\rangle \leq 0,
$$

thus,

$$
\left\langle V_{C}(x)-V_{C}(\hat{y}), V_{C}(y)-V_{C}(\hat{y})\right\rangle \geq 0 .
$$

We also have,By Proposition 4.1

$$
\begin{aligned}
\|x-y\|^{2} & =\|x-\hat{y}-(y-\hat{y})\|^{2} \\
& =\|x-\hat{y}\|^{2}+\|y-\hat{y}\|^{2}-2\langle x-\hat{y}, y-\hat{y}\rangle,
\end{aligned}
$$

and, similarly,

$$
\begin{aligned}
\left\|V_{C}(x)-V_{C}(y)\right\|^{2}= & \left\|V_{C}(x)-V_{C}(\hat{y})-\left(V_{C}(y)-V_{C}(\hat{y})\right)\right\|^{2} \\
= & \left\|V_{C}(x)-V_{C}(\hat{y})\right\|^{2}+\left\|V_{C}(y)-V_{C}(\hat{y})\right\|^{2} \\
& -2\left\langle V_{C}(x)-V_{C}(\hat{y}), V_{C}(y)-V_{C}(\hat{y})\right\rangle .
\end{aligned}
$$

Using 25), 29), 30 and 32 in 33 and 34 we get

$$
\left\|V_{C}(x)-V_{C}(y)\right\| \leq\|x-y\|,
$$


which proves the nonexpansivity of $V_{C}$ in this case.

Case B2. With an argument similar to the argument at the beginning of Case B1 we can assume here that

$$
\left\|P_{Z}(y)-V_{C}(y)\right\| \leq\left\|P_{Z}(x)-V_{C}(x)\right\|
$$

To study this case we add three enlargements to the set $Z$, one with a width of $\left\|P_{Z}(y)-y\right\|$, the second with a width of $\left\|P_{Z}(x)-V_{C}(x)\right\|$ and the third with a width of $\left\|P_{Z}(y)-V_{C}(y)\right\|$. We denote the intersection point between the line through $x$ and $P_{Z}(x)$ with the boundary of the first enlargement by $\hat{y}$. Therefore, the intersection of the above mentioned line with the boundary of the third enlargement is exactly $V_{C}(\hat{y})$. See Figure 4 , where the dashed lines are the enlargements.

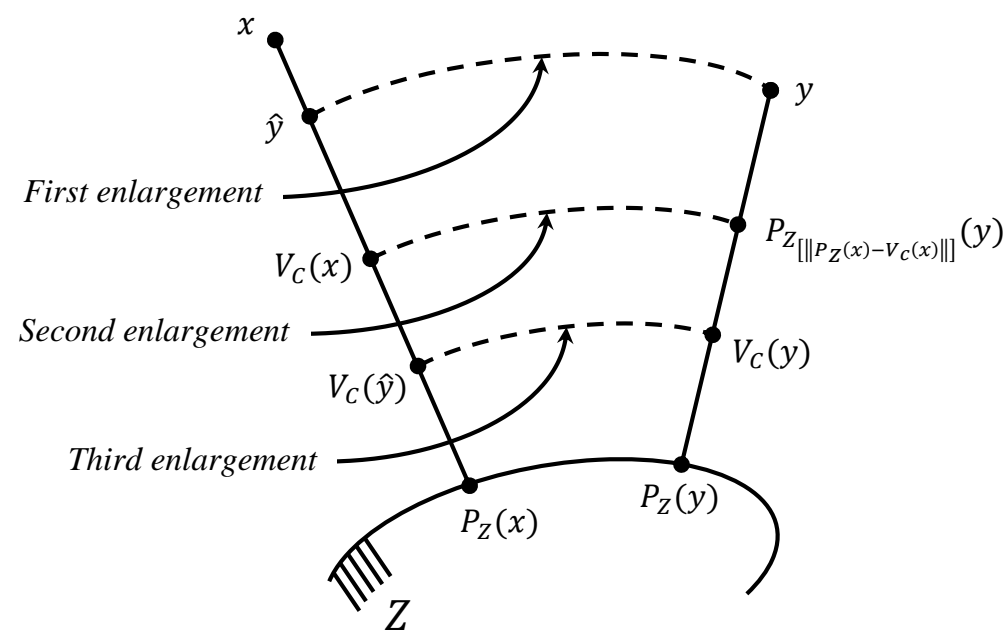

Fig. 4 Case B2 in the proof of Proposition 4.2

Now, using a reasoning similar to that in $24-25$, we calculate

$$
\begin{aligned}
\left\|V_{C}(\hat{y})-V_{C}(x)\right\| & =\left\|P_{Z}(x)-V_{C}(x)\right\|-\left\|P_{Z}(x)-V_{C}(\hat{y})\right\| \\
& =\beta(a-b)\left(1-\frac{\tau}{2}\left(1+\frac{1}{a b}\right)\right) \leq \beta(a-b)=\|x-\hat{y}\| .
\end{aligned}
$$

The intersection point between the second enlargement and the line which passes through $y$ and $P_{Z}(y)$ is $P_{Z_{\left[\left\|P_{Z}(x)-V_{C}(x)\right\|\right]}}(y)$. By the nonexpansivity of the metric projection we have

$$
\left\|P_{Z_{\left[\left\|P_{Z}(x)-V_{C}(x)\right\|\right]}}(y)-V_{C}(x)\right\| \leq\|y-\hat{y}\|
$$


By the characterization of the metric projection, for $P_{Z_{\left[\left\|P_{Z}(x)-V_{C}(x)\right\|\right]}}(y)$ we have

$$
\left\langle y-P_{Z_{\left[\left\|P_{Z}(x)-V_{C}(x)\right\|\right]}}(y), V_{C}(x)-P_{Z_{\left[\left\|P_{Z}(x)-V_{C}(x)\right\|\right]}}(y)\right\rangle \leq 0,
$$

and so

$$
\left\langle V_{C}(y)-P_{Z_{\left[\left\|P_{Z}(x)-V_{C}(x)\right\|\right]}}(y), V_{C}(x)-P_{Z_{\left[\left\|P_{Z}(x)-V_{C}(x)\right\|\right]}}(y)\right\rangle \geq 0 .
$$

Now by (30), 37), 38, 40, by using similar calculations as in (33), and by replacing $V_{C}(\hat{y})$ by $P_{Z_{\left[\left\|P_{Z}(x)-V_{C}(x)\right\|\right]}}(y)$ in 34 we obtain 35 , namely, the nonexpansivity of $V_{C}$.

Case B3. With an argument similar to the argument at the beginning of Case B1 we can assume here that

$$
\left\|P_{Z}(y)-y\right\| \leq\left\|P_{Z}(x)-V_{C}(x)\right\| .
$$

Consult Figure 5 . The points $x, y, P_{Z}(x), P_{Z}(y), V_{C}(x)$ and $V_{C}(y)$ depict the situation for this case. To study this case we add three enlargements to the set $Z$, one with a width of $\left\|P_{Z}(x)-x\right\|$, the second with a width of $\left\|P_{Z}(y)-y\right\|$ and the third with a width of $\left\|P_{Z}(y)-V_{C}(y)\right\|$. We denote the intersection between the line through $x$ and $P_{Z}(x)$ and the boundary of the second enlargement by $\hat{y}$. Therefore, as argued earlier, the intersection of this line with the boundary of the third enlargement is precisely $V_{C}(\hat{y})$. We denote the intersection between the line through $y$ and $P_{Z}(y)$ with the boundary of the first enlargement by $\hat{x}$.

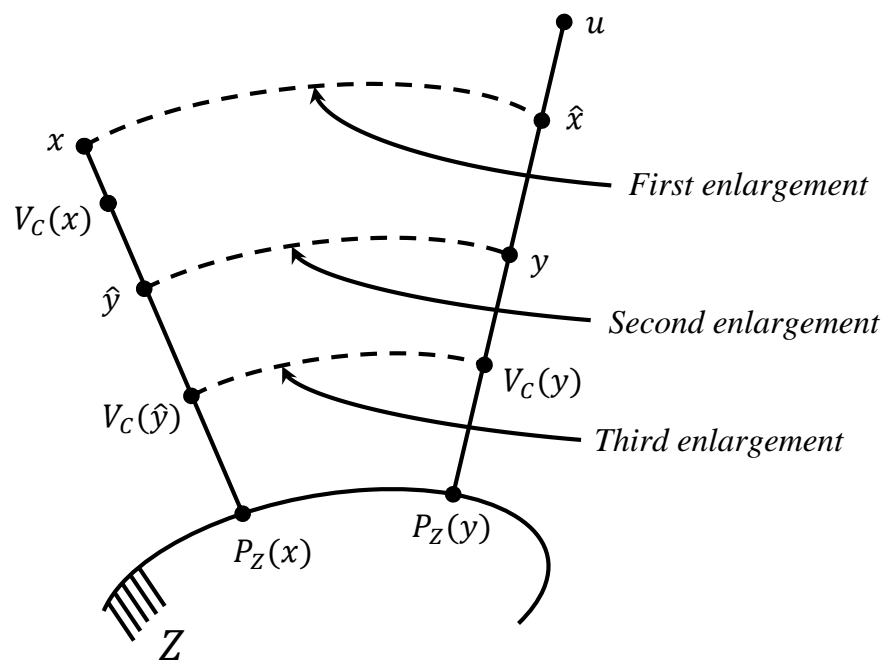

Fig. 5 Case B3 in the proof of Proposition 4.2 
Now we calculate

$$
\begin{aligned}
\left\|x-V_{C}(y)\right\|^{2}= & \left\|x-V_{C}(\hat{y})-\left(V_{C}(y)-V_{C}(\hat{y})\right)\right\|^{2} \\
= & \left\|x-V_{C}(\hat{y})\right\|^{2}+\left\|V_{C}(y)-V_{C}(\hat{y})\right\|^{2} \\
& -2\left\langle x-V_{C}(\hat{y}), V_{C}(y)-V_{C}(\hat{y})\right\rangle .
\end{aligned}
$$

Calculating the left-hand side of 42 a bit differently we may write

$$
\begin{aligned}
\left\|x-V_{C}(y)\right\|^{2} & =\left\|x-\hat{x}-\left(V_{C}(y)-\hat{x}\right)\right\|^{2} \\
& =\|x-\hat{x}\|^{2}+\left\|V_{C}(y)-\hat{x}\right\|^{2}-2\left\langle x-\hat{x}, V_{C}(y)-\hat{x}\right\rangle .
\end{aligned}
$$

Finally, we write

$$
\begin{aligned}
\|x-y\|^{2} & =\|x-\hat{x}-(y-\hat{x})\|^{2} \\
& =\|x-\hat{x}\|^{2}+\|y-\hat{x}\|^{2}-2\langle x-\hat{x}, y-\hat{x}\rangle .
\end{aligned}
$$

By subtracting 34 from 42, by subtracting 44 from 43, and then by subtracting the second result from the first and because

$$
\left\|x-V_{C}(\hat{y})\right\|^{2}=\left\|V_{C}(y)-\hat{x}\right\|^{2},
$$

we obtain,

$$
\begin{aligned}
\|x-y\|^{2}-\left\|V_{C}(x)-V_{C}(y)\right\|^{2}= & \|y-\hat{x}\|^{2}-\left\|V_{C}(x)-V_{C}(\hat{y})\right\|^{2} \\
& +2\left\langle V_{C}(x)-V_{C}(\hat{y}), V_{C}(y)-V_{C}(\hat{y})\right\rangle \\
& -2\left\langle x-V_{C}(\hat{y}), V_{C}(y)-V_{C}(\hat{y})\right\rangle \\
& +2\left\langle x-\hat{x}, V_{C}(y)-\hat{x}\right\rangle-2\langle x-\hat{x}, y-\hat{x}\rangle .
\end{aligned}
$$

Along the line through $x$ and $P_{Z}(x)$ we have, by using 19 ,

$$
\begin{aligned}
\left\|V_{C}(\hat{y})-V_{C}(x)\right\| & =\left\|V_{C}(\hat{y})-\hat{y}\right\|+\left\|\hat{y}-V_{C}(x)\right\| \\
& =\left\|V_{C}(y)-y\right\|+\left\|\hat{y}-V_{C}(x)\right\| \\
& \leq\left\|V_{C}(x)-x\right\|+\left\|\hat{y}-V_{C}(x)\right\| \\
& =\|\hat{y}-x\| \\
& =\|y-\hat{x}\| .
\end{aligned}
$$


By the linearity of the inner product and by using the characterization of the metric projection we have

$$
\begin{aligned}
\left\langle x-V_{C}(\hat{y}), V_{C}(y)-V_{C}(\hat{y})\right\rangle & \\
= & \left\langle x-V_{C}(x), V_{C}(y)-V_{C}(\hat{y})\right\rangle \\
& +\left\langle V_{C}(x)-V_{C}(\hat{y}), V_{C}(y)-V_{C}(\hat{y})\right\rangle \\
\leq & \left\langle V_{C}(x)-V_{C}(\hat{y}), V_{C}(y)-V_{C}(\hat{y})\right\rangle .
\end{aligned}
$$

Finally, let $u$ be a point on the line through $y$ and $P_{Z}(y)$ such that

$$
P_{Z_{\left[\left\|P_{Z}(y)-\hat{x}\right\|\right]}}(u)=\hat{x}
$$

By the characterization of the metric projection we have

$$
\langle x-\hat{x}, u-\hat{x}\rangle \leq 0
$$

so,

$$
\langle y-\hat{x}, x-\hat{x}\rangle \geq 0 \text {. }
$$

By the linearity of the inner product and by 51 we have

$$
\begin{aligned}
\left\langle V_{C}(y)-\hat{x}, x-\hat{x}\right\rangle & =\left\langle V_{C}(y)-y, x-\hat{x}\right\rangle+\langle y-\hat{x}, x-\hat{x}\rangle \\
& \geq\langle y-\hat{x}, x-\hat{x}\rangle .
\end{aligned}
$$

Using (47), 48, and 52 in 46 , we have

$$
\left\|V_{C}(x)-V_{C}(y)\right\| \leq\|x-y\|
$$

By 19 and considerations as in Cases B1 and B2 we get the nonexpansivity of $V_{C}$, and the proof is complete.

Possibility C: The first line of 10 holds for $y$, i.e., $V_{C}(y)=y$, and the second line of 10 holds for $x$.

Now we discuss the situation

$$
\begin{aligned}
& d_{Z}(x)>\beta, \\
& d_{Z}(y) \leq \beta,
\end{aligned}
$$

and repeat 16 and (17) but this time for some real $0<b \leq 1<a$. 
Case C1. With an argument similar to the argument at the beginning of Case B1 we can assume here that

$$
\left\|P_{Z}(x)-V_{C}(x)\right\| \geq\left\|P_{Z}(y)-y\right\| .
$$

To study this case we add two enlargements to the set $Z$, one with a width of $\left\|P_{Z}(y)-y\right\|$ and the other with a width of $\left\|P_{Z}(x)-V_{C}(x)\right\|$. We denote the intersection point of the line through $x$ and $P_{Z}(x)$ with the boundary of the first enlargement by $\hat{y}$, and with the boundary of the second enlargement by $V_{C}(x)$. We also denote the intersection point of the line through $y$ and $P_{Z}(y)$ with the boundary of the second enlargement by $V_{C}(\hat{x})$.

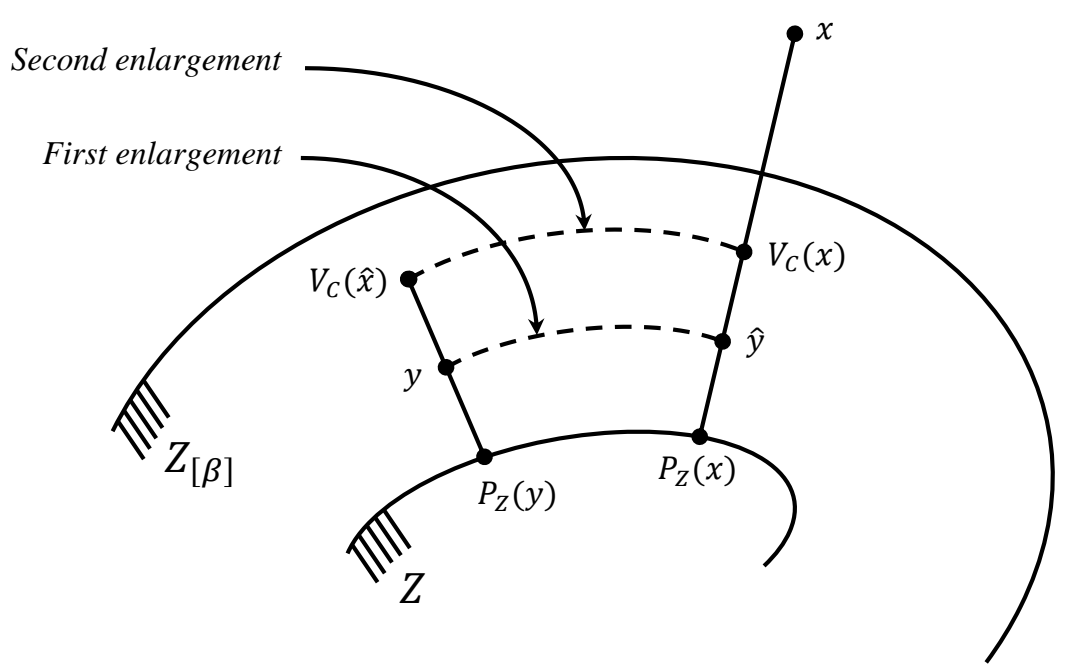

Fig. 6 Case $\mathrm{C} 1$ in the proof of Proposition 4.2

Now we have

$$
\|x-\hat{y}\| \geq\left\|V_{C}(x)-\hat{y}\right\|
$$

We also have

$$
\begin{aligned}
\left\|V_{C}(x)-V_{C}(y)\right\|^{2}= & \left\|V_{C}(x)-y\right\|^{2} \\
= & \left\|V_{C}(x)-\hat{y}-(y-\hat{y})\right\|^{2} \\
= & \left\|V_{C}(x)-\hat{y}\right\|^{2}+\|y-\hat{y}\|^{2} \\
& -2\left\langle V_{C}(x)-\hat{y}, y-\hat{y}\right\rangle .
\end{aligned}
$$

The characterization of the metric projection for $\hat{y}=P_{Z_{\left[\left\|P_{Z}(y)-y\right\|\right]}}(x)$ allows us to reuse $30 p$ and also yields

$$
\left\langle V_{C}(x)-\hat{y}, y-\hat{y}\right\rangle \leq 0 .
$$


Using (57), (58), 30, (59) and (33) proves the nonexpansivity of $V_{C}$ in this case.

Case C2. With an argument similar to the argument at the beginning of Case B1 we can assume here that

$$
\left\|P_{Z}(x)-V_{C}(x)\right\|<\left\|P_{Z}(y)-y\right\| .
$$

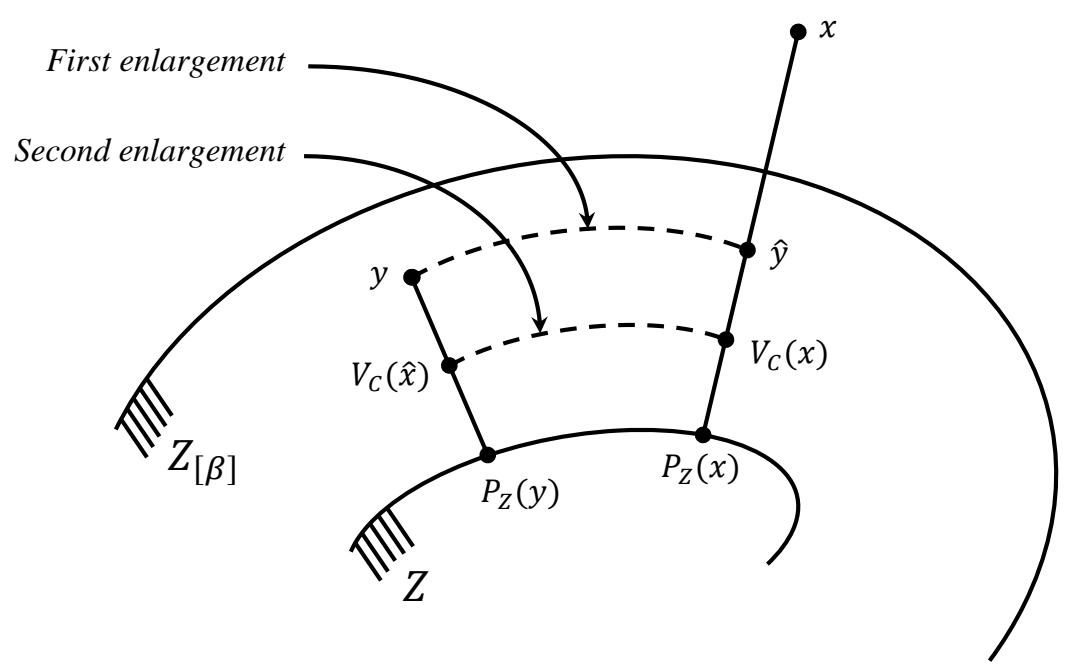

Fig. 7 Case $\mathrm{C} 2$ in the proof of Proposition 4.2

To study this case we repeat the addition of two enlargements exactly as described for Case C1 above. The only difference will be (see Figure 7) that the two enlargements have changed their positions.

The calculation of 22 in Case B1 holds again here verbatim. Now the following holds (recall that $0<b \leq 1<a$.)

$$
\begin{aligned}
\left\|V_{C}(x)-\hat{y}\right\| & =\left\|P_{Z}(y)-y\right\|-\left\|P_{Z}(y)-V_{C}(\hat{x})\right\| \\
& =\left\|P_{Z}(y)-y\right\|-\left(\left\|P_{Z}(x)-x\right\|-\left\|V_{C}(x)-x\right\|\right) \\
& =b \beta-\left(a \beta-\left|\frac{\tau}{2}\left(1-\left(\frac{\beta}{a \beta}\right)^{2}\right)\right|\left\|P_{Z}(x)-x\right\|\right) \\
& =\frac{\tau}{2}\left(1-\frac{1}{a^{2}}\right) a \beta-(a-b) \beta .
\end{aligned}
$$

By subtracting (61) from 22 we obtain

$$
\|x-\hat{y}\|-\left\|V_{C}(x)-\hat{y}\right\|=2(a-b) \beta-\frac{\tau}{2}\left(1-\frac{1}{a^{2}}\right) a \beta .
$$


Now we calculate the ratio

$$
\frac{2(a-b) \beta}{\frac{\tau}{2}\left(1-\frac{1}{a^{2}}\right) a \beta}=\frac{2}{\tau} \frac{a-b}{a-1} 2 \frac{a}{a+1} .
$$

Since $0<\tau<2$ and $0<b \leq 1$ we have $\frac{2}{\tau}>1$ and $\frac{a-b}{a-1} \geq 1$ and we have $\frac{1}{2}<\frac{a}{a+1}<1$, so that $1<2 \frac{a}{a+1}<2$, and then the ratio in 63 is greater than 1 , so the right side of 62 is greater than 0 and hence

$$
\|x-\hat{y}\|>\left\|V_{C}(x)-\hat{y}\right\|
$$

Reusing 30 we have

$$
\left\langle V_{C}(x)-\hat{y}, y-\hat{y}\right\rangle \geq 0 \text {. }
$$

Now by (33), (30), (58), 64) and $(65)$ proves the nonexpansivity of $V_{C}$ in this case.

Another important feature of the valiant operator is the following.

Proposition 4.3 Let $Z$ be a nonempty closed convex subset of $\mathcal{H}$, let $\beta \in \mathbb{R}, \beta>0$. If $C:=Z_{[\beta]}$ then the valiant projector $V_{C}$ has the property

$$
\operatorname{Fix} V_{Z_{[\beta]}}=Z_{[\beta]} .
$$

Proof If $x \in C$ then $d_{Z}(x) \leq \beta$ and so $V_{C}(x)=x$. Therefore, $x \in \operatorname{Fix} V_{C}$. If $x \in \operatorname{Fix} V_{C}$ then $V_{C}(x)=x$. If the first line of 10 holds then $x \in C$. If the second line of 10 holds then

$$
x=x+\frac{\tau}{2}\left(1-\left(\frac{\beta}{d_{Z}(x)}\right)^{2}\right)\left(P_{Z}(x)-x\right),
$$

which leads to either $\beta=d_{Z}(x)$ or $P_{Z}(x)=x$, implying in both cases that $x \in C$. Therefore, if $x \in$ $\operatorname{Fix} V_{C}(x)$ then $x \in C$.

\section{The Valiant Projections Method}

Now we are ready to present our algorithm that employs valiant projections and prove its convergence. A sequence $\left\{i_{k}\right\}_{k=0}^{\infty}$ of indices is called a cyclic control sequence on $I:=\{1,2, \ldots, m\}$ if $i_{k}=k(\bmod m)+1$.

Let $Z_{1}, Z_{2}, \ldots, Z_{m} \subseteq \mathcal{H}$ be nonempty closed convex sets and $C_{i}=\left(Z_{i}\right)_{\left[\beta_{i}\right]}$ their enlargements with $\beta_{i}>0$ for all $i \in I$. Assume that $\bigcap_{i \in I} C_{i} \neq \varnothing$. 


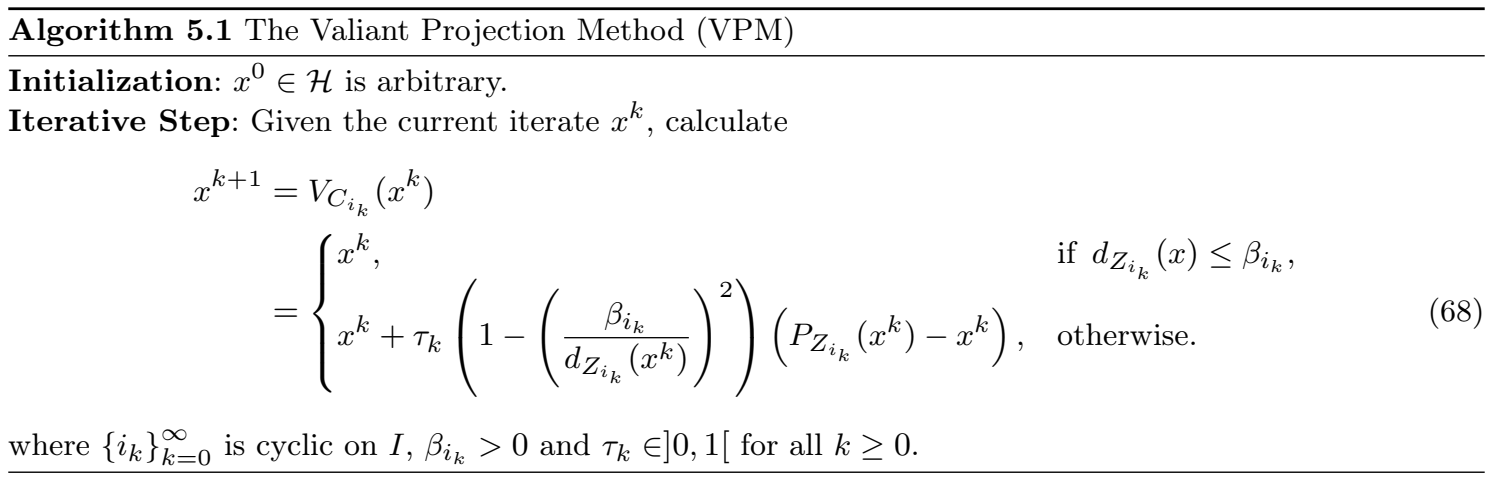

Theorem 5.1 Let $Z_{1}, Z_{2}, \ldots, Z_{m} \subseteq \mathcal{H}$ be nonempty closed convex sets and $C_{i}=\left(Z_{i}\right)_{\left[\beta_{i}\right]}$ their enlargements with $\beta_{i}>0$, for all $i \in I$. Assume that $\bigcap_{i \in I} C_{i} \neq \varnothing$. Any sequence $\left\{x^{k}\right\}_{k=0}^{\infty}$, generated by Algorithm 5.1. converges weakly to a point $x^{*} \in \bigcap_{i \in I} C_{i}$.

Proof We wish to apply Theorem 2.5 and to this end we show that all assumptions of that theorem hold here. Let $X \subseteq \mathcal{H}$ be a nonempty closed convex subset, we define an operator $S: X \rightarrow X$ by

$$
S:=\prod_{i=1}^{m} V_{C_{i}}=V_{C_{m}} V_{C_{m-1}} \cdots V_{C_{1}}
$$

By Proposition 4.1, each $V_{C_{i}}$ is SQNE, so, by Proposition 2.2(iii), it is sQNE. By Proposition 4.3 we have

$$
\bigcap_{i=1}^{m} \operatorname{Fix} V_{C_{i}}=\bigcap_{i=1}^{m} C_{i} \neq \varnothing .
$$

Using Theorem 2.1 (observe that this theorem dictates the use of the cyclic control in our algorithm) with $U_{i}$ as the valiant operators $V_{C_{i}}$, and applying Proposition 2.2 (i), we get

$$
\operatorname{Fix} S=\bigcap_{i=1}^{m} \operatorname{Fix} V_{C_{i}} \text {. }
$$

Thus, by Remark 2.2, the operator $S$ is SQNE and so, by Theorem 2.2, it is asymptotically regular. From Proposition 4.2, $V_{C_{i}}$ is NE, and by, Remark 2.1(ii), $S$ is NE and has a fixed point according to 70 and (71). Using the demiclosedness principle embodied in Theorem 2.4 and Definition 2.5 for the operator $S$, the operator $S-$ Id is demiclosed at 0 .

Since the iterative process of the algorithm consists of repeated applications of the valiant operator we show next that $\left\{V_{C_{i_{k}}}\right\}_{k=0}^{\infty}$ is an asymptotically regular sequence of QNE operators. Since $V_{C_{i}}$ is SQNE we have, by Definition 2.3 (iv) with $\alpha=1$,

$$
\left\|V_{C_{i_{k}}}\left(x^{k}\right)-z\right\|^{2} \leq\left\|x^{k}-z\right\|^{2}-\left\|V_{C_{i_{k}}}\left(x^{k}\right)-x^{k}\right\|^{2} \text {, for every } z \in \operatorname{Fix} V_{C_{i_{k}}},
$$


which guarantees that

$$
\left\|x^{k+1}-z\right\|^{2} \leq\left\|x^{k}-z\right\|^{2}-\left\|x^{k+1}-x^{k}\right\|^{2} \text {, for every } z \in \bigcap_{k=0}^{\infty} \operatorname{Fix} V_{C_{i_{k}}}
$$

Consequently, $\left\{x^{k}\right\}$ is Fejér-monotone with respect to $\bigcap_{k=0}^{\infty} \operatorname{Fix} V_{C_{i_{k}}}$ thus it is bounded. Therefore, $\left\{\left\|x^{k}-z\right\|\right\}_{k=0}^{\infty}$ is monotonically decreasing thus convergent, which yields

$$
\lim _{k \rightarrow \infty}\left\|x^{k+1}-x^{k}\right\|=0
$$

According to Definition $2.6\left\{V_{C_{i_{k}}}\right\}_{k=0}^{\infty}$ is an asymptotically regular sequence of QNE operators. Finally, to justify 6 we compute the following limit using the triangle inequality and 74 .

$$
\begin{aligned}
& \lim _{k}\left\|S\left(x^{k}\right)-x^{k}\right\| \\
& =\lim _{k}\left\|\prod_{i=1}^{m} V_{C_{i}}\left(x^{k}\right)-x^{k}\right\| \\
& =\lim _{k}\left\|x^{k+m}-x^{k}\right\| \\
& =\lim _{k}\left\|x^{k+m}-x^{k-1+m}+x^{k-1+m}-x^{k-2+m}+x^{k-2+m} \cdots-x^{k+1}+x^{k+1}-x^{k}\right\| \\
& \leq \lim _{k}\left(\left\|x^{k+m}-x^{k-1+m}\right\|+\left\|x^{k-1+m}-x^{k-2+m}\right\|+\cdots+\left\|x^{k+1}-x^{k}\right\|\right) \\
& =\lim _{k}\left\|x^{k+m}-x^{k-1+m}\right\|+\lim _{k}\left\|x^{k-1+m}-x^{k-2+m}\right\|+\cdots+\lim _{k}\left\|x^{k+1}-x^{k}\right\| \\
& =0 .
\end{aligned}
$$

We have proved that all the assumptions of Theorem 2.5 are satisfied. Therefore, $\left\{x^{k}\right\}_{k=0}^{\infty}$ converges weakly to a point $x^{*} \in \operatorname{Fix} S$ and, by 70 and $(71), x^{*} \in \bigcap_{i=1}^{m} C_{i}$.

\section{Conclusions}

In Table 1 we depict features and relationships between the algorithmic operators which grew out from Goffin's principle. The idea of enlargements led to the extensions of the algorithms ART3 and ARM to handle convex sets. As stated in [18, Theorems 11 and 14], convergence of the method of cyclic intrepid projections (CycIP), see, [18, Algorithm 9] is guaranteed if the interior of the intersection of the sets is not empty. In the present work with valiant operators this condition is not required for the convergence of our VPM algorithm. 
Table 1 Approaches to Goffin's principle

\begin{tabular}{|c|c|c|c|}
\hline & \multicolumn{2}{|c|}{ "Far-Near" iterative step determination } & $\begin{array}{c}\text { “Continuous" } \\
\text { iterative step } \\
\text { determination }\end{array}$ \\
\hline The linear case & \multicolumn{2}{|c|}{ The ART3 operator (Herman 1975) } & $\begin{array}{c}\text { The ARM operator } \\
\text { (Censor 1985) }\end{array}$ \\
\hline $\begin{array}{l}\text { The general } \\
\text { convex case }\end{array}$ & $\begin{array}{c}\text { The BIK intrepid } \\
\text { projection operator } \\
\text { (Bauschke et al. } \\
\text { 2014) }\end{array}$ & $\begin{array}{c}\text { The geometric } \\
\text { intrepid projection } \\
\text { operator (present } \\
\text { work) }\end{array}$ & $\begin{array}{c}\text { The valiant } \\
\text { projection operator } \\
\text { (present work) }\end{array}$ \\
\hline
\end{tabular}

Acknowledgements We thank Tommy Elfving for reading several parts of this paper and making enlightening comments. We are indebted to the reviewers and to the Editor-in-Chief Franco Giannessi for their insightful and constructive comments that helped us improve the paper. This work was supported by Research Grant No. 2013003 of the United States-Israel Binational Science Foundation (BSF).

\section{References}

1. H.H. Bauschke and J.M. Borwein. On projection algorithms for solving convex feasibility problems. SIAM Review, 38:367-426, 1996.

2. Y. Censor and A. Cegielski. Projection methods: an annotated bibliography of books and reviews. Optimization, 64:2343-2358, 2015.

3. H.H. Bauschke and V. Koch. Projection methods: Swiss army knives for solving feasibility and best approximation problems with halfspaces. Contemporary Mathematics, 636:1-40, 2015.

4. H.H. Bauschke, V. Koch, and H. Phan. Stadium norm and Douglas-Rachford splitting: a new approach to road design optimization. Operations Research, 64:201-218, 2016.

5. F.J. Aragón Artacho, J.M. Borwein, and M.K. Tam. Douglas-Rachford feasibility methods for matrix completion problems. The ANZIAM Journal, 55:299-326, 2014.

6. J.M. Borwein and M.K. Tam. Reflection methods for inverse problems with application to protein conformation determination. In Generalized Nash Equilibrium Problems, Bilevel programming and MPEC. Springer, New Delhi, 2012 .

7. F.J. Aragón Artacho, J.M. Borwein, and M.K. Tam. Recent results on Douglas-Rachford methods for combinatorial optimization problems. Journal of Optimization Theory and Applications, 163:1-30, 2014.

8. Y. Censor and S.A. Zenios. Parallel Optimization: Theory, Algorithm, and Applications. Oxford University Press, New York, NY, USA, 1997.

9. A. Cegielski. Iterative Methods for Fixed Point Problems in Hilbert Spaces. Lecture Notes in Mathematics 2057, Springer-Verlag, Berlin, Heidelberg, Germany, 2012.

10. H.H. Bauschke and P.L. Combettes. Convex Analysis and Monotone Operator Theory in Hilbert Spaces. Springer, New York, NY, USA, 2011.

11. S. Agmon. The relaxation method for linear inequalities. Canadian Journal of Mathematics, 6:382-392, 1954. 
12. T.S. Motzkin and I.J. Schoenberg. The relaxation method for linear inequalities. Canadian Journal of Mathematics, 6:393-404, 1954.

13. J.L. Goffin. On the finite convergence of the relaxation method for solving systems of inequalities. Operation Research Center, Report ORC 71-36, Ph.D. thesis, University of California, Berkeley, 1971.

14. G.T. Herman. A relaxation method for reconstructing objects from noisy x-rays. Mathematical Programming, $8: 1-19,1975$.

15. Y. Censor. An automatic relaxation method for solving interval linear inequalities. Journal of Mathematical Analysis and Applications, 106:19-25, 1985.

16. Y. Censor and G.T. Herman. On some optimization techniques in image reconstruction from projections. Applied Numerical Mathematics, 3:365-391, 1987.

17. Y. Censor. Parallel application of block-iterative methods in medical imaging and radiation therapy. Mathematical Programming, 42:307-325, 1988.

18. H.H. Bauschke, F. Iorio, and V. Koch. The method of cyclic intrepid projections: convergence analysis and numerical experiments. In M. Wakayama, R.S. Anderssen, J. Cheng, Y. Fukumoto, R. McKibbin, K. Polthier, T. Takagi, and K-C. Toh, editors, The Impact of Applications on Mathematics, pages 187-200. Springer, 2014. 